\title{
The use of streptomycin resistance and chlorophyll deficiency for selection of somatic hybrids between Nicotiana tabacum and $N$. rustica
}

\author{
Seiichi Toki and \\ Toshiaki Kameya
}

\author{
Institute for Agricultural Research, Tohoku \\ University, Sendai 980, Japan.
}

To prove the usefulness for somatic hybridisation of a Nicotiana tabacum L. double mutant (SA), which is both streptomycin resistant and chlorophyll deficient, protoplasts obtained from $\mathrm{SA}$ suspension cultures were fused via the dextran method with protoplasts isolated from $\mathbf{N}$. rustica $\mathbf{L}$. mesophyll cells. Prospective somatic hybrids were selected for streptomycin resistance and the ability to produce chlorophyll in regenerated plants. By using this selection system, green plants were recovered from 5 colonies. The hybrid nature of these plants was confirmed by morphological studies and isoelectric focusing of the Fraction 1 protein (RuBP carboxylase). The present results show evidence for the potential use of the double mutant in studies of somatic hybridisation.

\section{INTRODUCTION}

Recently, by using universal hybridisers, which have both positive and negative selection markers, some somatic hybrids have been obtained effectively (LoSchiavo et al., 1983; Pental et al., 1984; Ye and Widholm, 1986). In the preceding paper (Toki and Kameya, 1987), we reported production of a double mutant of Nicotiana tabacum (SA) carrying markers for streptomycin resistance and chlorophyll deficiency. Calli initiated from SA showed resistance to streptomycin, and the chlorophyll content in SA leaves was lower than that of the normal green plants. Therefore, it was expected that the double mutant SA could be useful in studies of somatic hybridisation. The present paper describes production of somatic hybrid plants between $N$. tabacum SA and $N$. rustica by using streptomycin resistance and chlorophyll deficiency for selection.

\section{MATERIALS AND METHODS}

\section{Plant materials}

A streptomycin resistant chlorophyll deficient mutant of Nicotiana tabacum L. (Toki and Kameya, 1987) and $N$. rustica L. were used for experimentation. A suspension culture of $N$. tabacum was prepared as described previously (Toki and Kameya, 1987).

\section{Isolation of protoplasts}

N. tabacum SA protoplasts were isolated from 16month-old suspension cultured cells by incubating in a solution containing 9 per cent $(\mathrm{W} / \mathrm{V})$ mannitol, $0 \cdot 1$ per cent $(\mathrm{W} / \mathrm{V}) \mathrm{CaCl}_{2} \cdot 2 \mathrm{H}_{2} \mathrm{O}, 0.5$ per cent $(\mathrm{W} / \mathrm{V})$ macerozyme $\mathrm{R} 10$ and 3 per cent $(\mathrm{W} / \mathrm{V})$ cellulase Onozuka R10 for 12 hours at $25^{\circ} \mathrm{C}$. $N$. rustica protoplasts were isolated from leaves by incubating in the same enzyme solution for 3 hours at $25^{\circ} \mathrm{C}$.

In both cases, protoplasts were filtered through a mesh $(56 \mu \mathrm{m})$, washed twice with a washing solution (containing 9 per cent $(\mathrm{W} / \mathrm{V})$ mannitol, $0 \cdot 1$ per cent $\mathrm{CaCl}_{2} \cdot 2 \mathrm{H}_{2} \mathrm{O}$ ), floated to the surface with $20 \%(\mathrm{~W} / \mathrm{V})$ sucrose, collected by centrifugation ( $80 \mathrm{~g}$ for 5 minutes) and resuspend in the washing solution.

\section{Protoplast fusion}

Protoplasts were fused according to the method of Kameya (1983) with minor modifications. One drop (about $0.2 \mathrm{ml}$ ) of fusion medium (containing 15 per cent $(\mathrm{W} / \mathrm{V})$ dextran and 6 per cent $(\mathrm{W} / \mathrm{V})$ $\mathrm{NaCl}$ ) was placed in a Petri dish and one drop of the protoplast suspension containing $N$. tabacum SA and $N$. rustica cells was placed on the fusion medium. Then 3 drops of fusion medium were added to the protoplast suspension and mixed gently by shaking the Petri dish. Fifteen minutes 
Table 1 Composition of culture media ${ }^{a}$

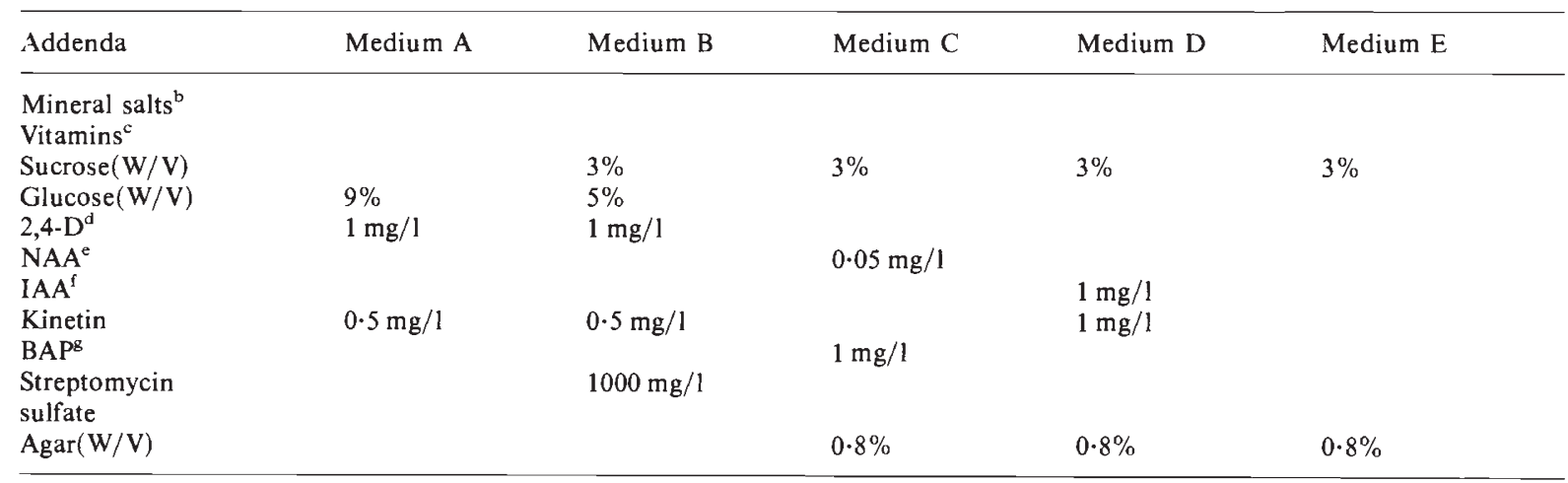

${ }^{\mathrm{a}} \mathrm{pH}$ was adjusted to $5 \cdot 7 .{ }^{\mathrm{b}}$ Murashige and Skoog (1962) formulation. ${ }^{\mathrm{c}}$ Uchimiya and Murashige (1974) formulation. ${ }^{\mathrm{d}} 2,4-$ dichlorophenoxyacetic acid. ${ }^{\mathrm{e}}$ indole-3-acetic acid. ${ }^{\mathrm{f}} \alpha$-naphthaleneacetic acid. ${ }^{\mathrm{g}}$ benzylaminopurine.

later one drop of $\mathrm{NaOH}-\mathrm{NaCl}$ solution (containing $0 \cdot 1 \mathrm{~N} \mathrm{NaOH}$ and 8 per cent $(\mathrm{W} / \mathrm{V}) \mathrm{NaCl}$ ) was added. After 15 minutes, $1 \mathrm{ml}$ of an elution medium $(5$ per cent $(\mathrm{W} / \mathrm{V})$ mannitol and 2 per cent $(\mathrm{W} / \mathrm{V})$ $\mathrm{CaCl}_{2} \cdot 2 \mathrm{H}_{2} \mathrm{O}$ ) was added six times at 5 minute intervals. The fused protoplasts were collected by centrifugation ( $80 \mathrm{~g}$ for 5 minutes).

\section{Culture of protoplasts and plant regeneration}

The fused protoplasts $\left(1 \times 10^{5}\right)$ were cultured in $4 \mathrm{ml}$ of protoplast culture medium A (table 1) in plastic Petri dishes at $25^{\circ} \mathrm{C}$. During the first 14 days, Petri dishes were incubated under continuous weak light $\left(0 \cdot 1 \mathrm{~W} / \mathrm{m}^{2}\right)$, and then transferred to strong light $\left(4 \mathrm{~W} / \mathrm{m}^{2}\right)$. After 10 days of culture, the cell suspension was diluted with an equal volume of the selection medium (medium $\mathrm{B}$, table 1), according to the selection scheme in fig. 1. After 20 days of culture, clusters consisting of 10-20 cells were mixed with equal volume of melted medium B containing 0.6 per cent agar, and placed in a Petri dish. After growth, calli about 1-2 mm in diameter, were transfered onto shooting medium (medium $\mathrm{C}$ or $\mathrm{D}$, table 1 ). Regenerated shoots were transferred onto a rooting medium (medium E).

\section{Analysis of fraction 1 protein}

The polypeptide composition of Fraction 1 protein (RuBP carboxylase) of regenerated plants was determined by the procedure of Hirai (1982).

\section{Streptomycin sensitivity of hybrid plant}

The callus of putative somatic hybrid was induced from leaves on MS (Murashige and Skoog, 1962) agar $(0 \cdot 8$ per cent $)$ medium with $1 \mathrm{mg} / 12,4-\mathrm{D}$ (2,4-dichlorophenoxyacetic acid) and $0.1 \mathrm{mg}$ Kinetin, then transferred onto the same medium containing $0-1000 \mathrm{mg} / 1$ streptomycin sulfate. The fresh weight was determined after 2 weeks.

\section{RESULTS}

\section{Selection of somatic hybrids}

After 1 month of culture, colonies (1-2 mm in diameter) appeared on the selection medium $B$ (table 1) which contained $750 \mathrm{mg} / 1$ streptomycin sulfate as final concentration. The colours of the colonies were white, yellowish-green, green as well as intermediate colours.

Twenty colonies were transferred to shooting medium C. Sixteen of them produced small calli (2-3 $\mathrm{mm}$ in diameter). From these 2 calli (called $\mathrm{H} 1$ and $\mathrm{H} 2$ ) several green plants were regenerated, 1 callus regenerated an albino plant.

Forty colonies were transferred to shooting medium D. Eighty per cent of them were yielded small calli, but only 1 callus regenerated an albino plant. Others were then transferred to shooting medium C. Of these, 3 calli (called $\mathrm{H} 3, \mathrm{H} 4$ and $\mathrm{H} 5$ ) regenerated several green plants.

The green plants regenerated from selected calli were expected to be somatic hybrid plants from the selection scheme in fig. 1. So we transferred these green plants to the hormone free rooting medium E.

The plants regenerated from $\mathrm{H} 2$ calli (H2P) were rooted easily on medium $\mathrm{E}$ and grew vigorously. The plants regnerated from other calli were rooted only occasionally. But 1 year after fusion treatment, at least one plant from each callus had been rooted. 


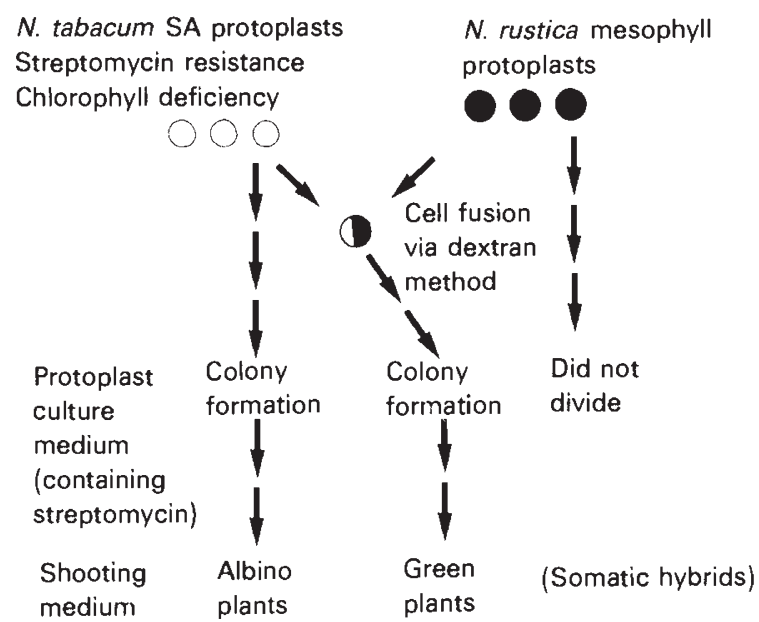

Figure 1 Selection scheme for hybrids between $N$. tabacum $\mathrm{SA}$ and $N$. rustica.

\section{Characterisation of hybrids}

Flower morphology of regenerated green plants were intermediate between $N$. tabacum and $N$. rustica for corolla length and width (fig. 2). While corollas of $N$. tabacum were pink and those of $N$. rustica were greenish-yellow, there were two types of plants among regenerated plants: one type had pinkish-yellow flowers, the other type, creamy-white. Pinkish-yellow flowers and creamy-white flowers were not found on an individual plant.

With respect to leaf morphology, $N$. tabacum lacks a petiole and possesses oval shaped leaves. $N$. rustica has a well formed petiole, egg shaped leaves and trichomes longer than that of $N$. tabacum. Regenerated green plants showed an intermediate morphology and had trichomes like a $N$. rustica but distribution of the trichomes was more random than that of $N$. rustica (fig. 3).

One plant of $\mathrm{H} 2 \mathrm{P}$ was analysed for the polypeptide composition of Fraction 1 protein (fig. 4). The large subunit polypeptides of this plant corresponded to the $N$. rustica type, indicating the presence of chloroplasts from $N$. rustica. The small subunit polypeptides of this plant contained both parental types of polypeptides; one being characteristic of the protein from $N$. rustica, one of that from $N$. tabacum and the third being common to both species. This result indicate that the nuclear genomes from both species were present in this plant. Also esterase isozyme patterns provided evidence of hybridity for H2P (data not shown).

\section{Streptomycin sensitivity of hybrid plant}

The callus derived from one of $\mathrm{H} 2 \mathrm{P}$ was sensitive to streptomycin like N. rustica callus (fig. 5). This indicates lack of $N$. tabacum chloroplasts which are resistant to streptomycin in this plant.

\section{DISCUSSION}

The colonies which appeared on the selection medium, containing $750 \mathrm{mg} / 1$ streptomycin sulfate, were expected to be $N$. tabacum colonies or somatic hybrid colonies. When we transferred some of these colonies to shooting medium, several albino plants and green plants were obtained. The latter were likely to be somatic hybrids. These plants expressed the morphology expected of a somatic hybrid and no evidence of unhybridised $N$. rustica was observed. Fraction 1 protein analysis of $\mathrm{H} 2 \mathrm{P}$ also showed evidence for hybridity.

Fraction 1 protein analysis of $\mathrm{H} 2 \mathrm{P}$ showed that the chloroplasts originated from $N$. rustica, and
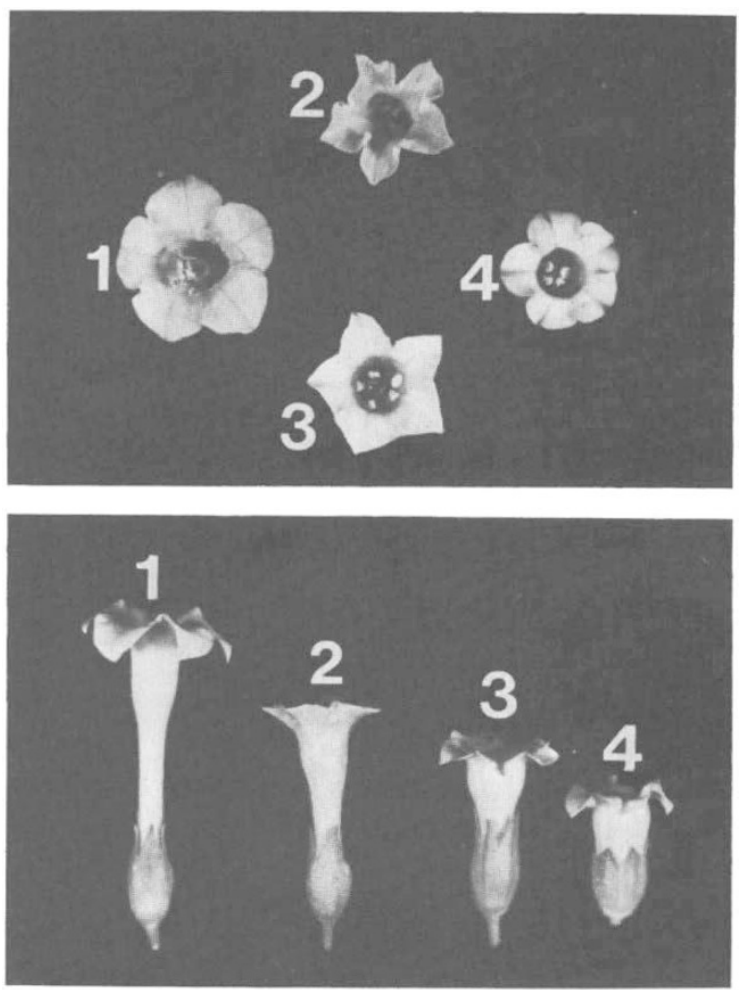

Figure 2 Flower morphology of somatic hybrids between $N$. tabacum SA and N. rustica, and parents. (1) N. tabacum $\mathrm{SA}$, (2) a somatic hybrid (pinkish-yellow flower), (3) a somatic hybrid (creamy-white flower), (4) N. rustica. 

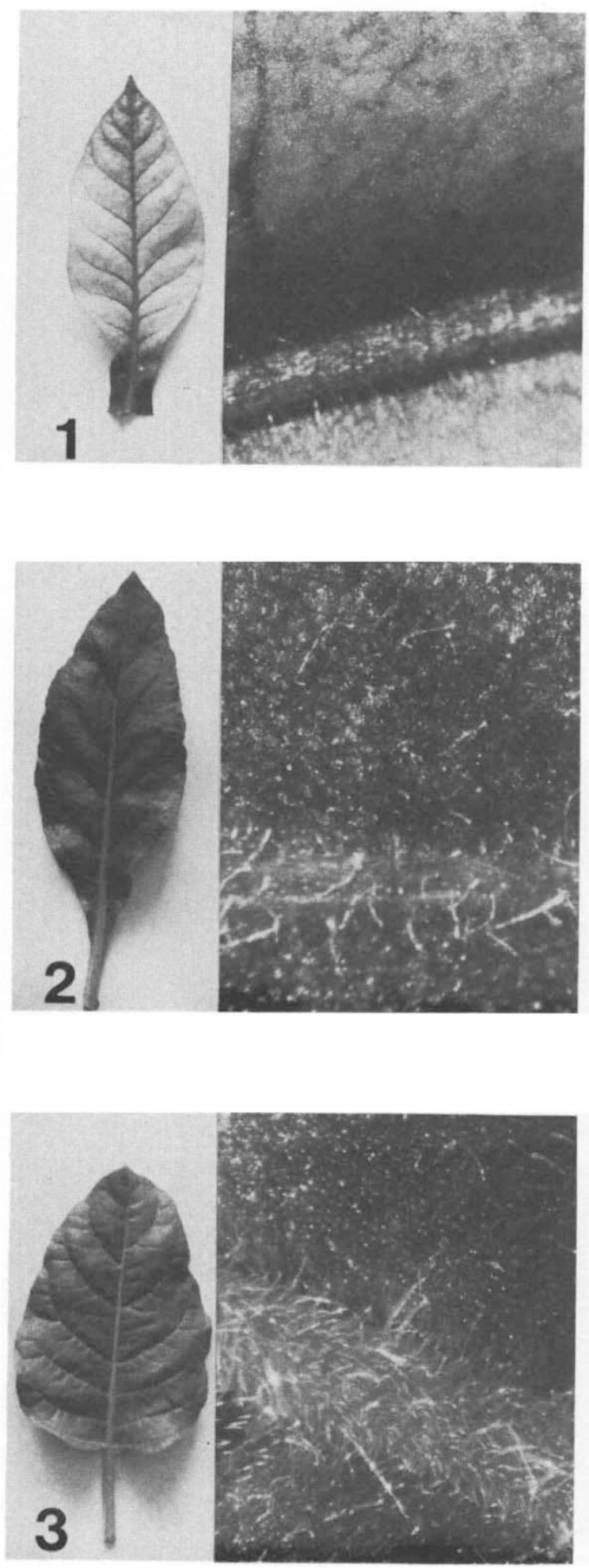

Figure 3 Leaf and trichomes on the leaf surface of a somatic hybrid between $N$. tabacum SA and $N$. rustica, and parents. (1) N. tabacum SA, (2) a somatic hybrid, (3) N. rustica.
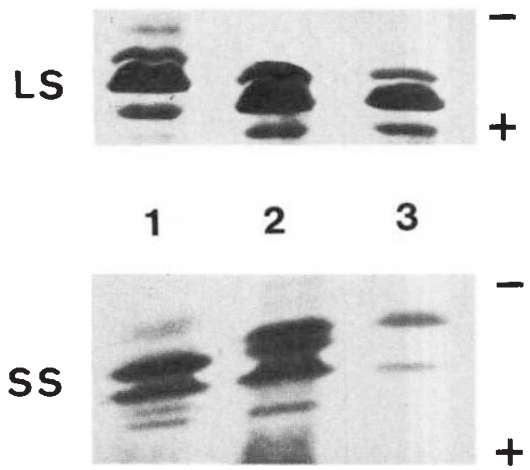

Figure 4 Large and small subunit polypeptides of Fraction 1 protein resolved by isoelectrofocusing polyacrylamide gel electrophoresis. (1) N. tabacum SA, (2) a somatic hybrid, (3) N. rustica. LS and SS denote large and small subunits of Fraction 1 protein, respectively.

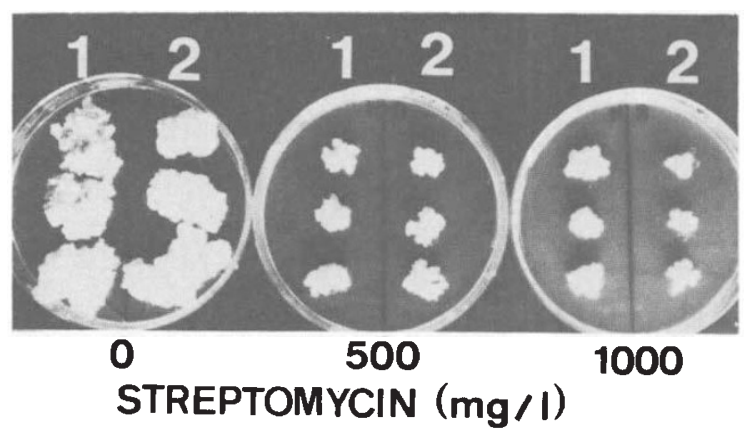

Figure 5 Streptomycin resistance test. (1) a somatic hybrid (H2P), (2) N. rustica.

callus obtained from $\mathrm{H} 2 \mathrm{P}$ showed the presence of streptomycin sensitive chloroplasts of $N$. rustica. Both results showed that streptomycin sensitive chloroplasts had not been eliminated completely by growth on selection medium. Similar results have been reported recently by Bourgin et al. (1986). The chloroplasts constitution of other hybrid plants are now being investigated.

Somatic hybrids between $N$. tabacum and $N$. rustica have been produced using selection systems for hybrids, such as callus morphology by Nagao (1978) and genetic complementation for chlorophyll synthesis by Douglas et al. (1981). However, these selection systems seem to be limited to combinations of parental plants.

Pental et al. (1984), however, obtained hybrids by using a nitrate reductase deficient, streptomycin resistant double mutant $\left(\mathrm{NR}^{-} \mathrm{SR}^{+}\right)$of $N$. tabacum. They selected hybrid colonies for nitrate reductase proficiency and streptomycin resistance, and then obtained hybrid plants on regeneration medium. 
In our experiment using the double mutant $N$. tabacum SA, somatic hybrids could be selected initially by culture on a medium containing streptomycin and then by the green pigmentation of the regenerated plants. So we can conclude that $N$. tabacum plants can be used as a universal hybridiser, like $\mathrm{NR}^{-} \mathrm{SR}^{+}$as established by Hamill et al. (1983), to hybridise with any streptomycin sensitive green plants.

Acknowledgements We thank Dr A. Hirai, Nagoya University, Japan, for demonstrating the Fraction 1 protein analysis.

\section{REFERENCES}

BouRGIN, J. P., MISSONIER, C. AND Goujaud, J. 1986. Direct selection of hybrids by streptomycin and valine resistance in tobacco. Theor. Appl. Genet., 72, 11-14.

DOUGlaS, G. C., KELLER, W. A. AND SETTERFIELD, G. 1981. Somatic hybridisation between Nicotiana rustica and $N$. tabacum. II. Protoplast fusion and selection and regeneration of hybrid plants. Can. J. Bot., 59, 220-227.

HAMILl, J. D., PENTAL, D., COCKING, E. C. AND MULleR, A. J. 1983. Production of a nitrate reductase deficient strep. tomycin resistant mutant of Nicotiana tabacum for somatic hybridisation studies. Heredity, 50, 197-200.

HIRAI, A. 1982. Isoelectrofocusing of non-carboxymethylated fraction I protein from green callus. Plant Sci. Lett., 25, $37-41$.

KAMEYA, T. 1983. Studies on plant cell fusion by dextran: Effects of $p \mathrm{H}$, inorganic salts and electrical stimulus. Cytologia, 48, 873-878.

LOSCHIVo, F., GIVINAZZo, G. AND TERZI, M. 1983. 8Azaguanine resistant carrot cell mutants and their use as universal hybridisers. Mol. Gen. Genet., 192, 326-329.

MURASHIGE, T. AND SKOOG, F. 1962. A revised medium for rapid growth and bio assays with tobacco tissue cultures. Physiol. Plant., 15, 473-497.

NAGAO, T. 1978. Somatic hybridisation by fusion of protoplasts. 1. The combination of Nicotiana tabacum and Nicotiana rustica. Japan J. Crop Sci., 47, 491-498.

PENTAL, D., HAMILl, J. D. AND COCKING, E. C. 1984. Somatic hybridisation using a double mutant of Nicotiana tabacum. Heredity, 53, 79-83.

TOKI, S. AND KAMEYA, T. 1987. Production of a chlorophyll deficient streptomycin resistant mutant of Nicotiana tabacum for somatic hybridisation studies. Heredity, 59, 223-226.

UCHIMIYA, H. AND MURASHIGE, T. 1974. Evaluation of parameters in the isolation of viable protoplasts from cultured tobacco cells. Plant Physiol., 54, 936-944.

YE, J. AND WIDHOLM, J. M. 1986. Somatic hybridisation using an universal hybridiser of Nicotiana plumbaginifolia. VI IAPTC, 389. 